\title{
STRUCTURAL AND MAGNETIC PROPERTIES OF $\mathrm{Dy}(\mathrm{Fe}, \mathrm{V}, \mathrm{Si})_{12} \mathrm{COMPOUNDS}$
}

\author{
Z. Drzazga, A. Winiarska, A. Chrobak \\ Institute of Plyysics, University of Silesia \\ Uniwersytecka 4, 40-007 Katowice, Poland \\ AND D. KACZOROWSKI \\ Institute of Low Temperature and Structure Research \\ Polish Academy of Sciences \\ P.O. Box 937, Wrocław 2, Poland
}

\begin{abstract}
An influence of $\mathrm{Si}$ on the structural and magnetic properties of $\mathrm{Dy}(\mathrm{Fe}, \mathrm{V}, \mathrm{Si})_{12}$ compounds is studied. $\mathrm{X}$-ray diffraction shows that these materials crystallise in the tetragonal $\mathrm{ThMn}_{12}$-type structure. An increase in the saturation magnetization, the Curie temperatures and magnetocrystalline anisotropy in the Si substitutional systems is observed when compared with $\mathrm{DyFe}_{12-x} \mathrm{~V}_{x}$. The magnetic properties depend on the preferential site occupation and concentration of $\mathrm{Si}$ and $\mathrm{V}$ atoms in these compounds.
\end{abstract}

PACS numbers: $61.10 .-\mathrm{i}, 75.30 . \mathrm{Cr}, 75.30 . \mathrm{Gw}, 75.50 . \mathrm{Gg}$

\section{Introduction}

Iron-rich intermetallic compounds of the $\mathrm{ThMn}_{12}$-type structure have been proposed as inexpensive alternatives for permanent magnetic materials. It is known that the rare earths and iron do not form the binary $\mathrm{RFe}_{12}$ phase, a third element is necessary to stabilize the tetragonal $\mathrm{ThMn}_{12}$-type structure [1]. These compounds have the composition $\mathrm{RFe}_{12-x} \mathrm{M}_{x}$, in which $\mathrm{M}$ represents a nonmagnetic $3 d$ element, for instance $\mathrm{Ti}, \mathrm{V}, \mathrm{Cu}$ or metalloid Si. Apart from the R elements and $\mathbf{3 d}$ elements, which mainly determine the magnetic properties of the compounds, also stabilizing elements contribute to the magnetic properties. The aim of this work is to study the influence of the substitution of $\mathrm{Si}$ instead of $\mathrm{V}$ on the magnetic properties of $\mathrm{DyFe}_{12-x} \mathrm{M}_{x}$ compounds.

\section{Experimental}

Samples of nominal composition $\mathrm{DyFe}_{10} \mathrm{~V}_{2-x} \mathrm{Si}_{x}, \mathrm{DyFe}_{9} \mathrm{~V}_{3-x} \mathrm{Si}_{x}$ and DyFe $\mathrm{V}_{4-x} \mathrm{Si}_{x}(x=0.05,1.0,1.5,2.0)$ were prepared by arc-melting. The samples were prepared in the same way as those described in [2].

The structure and homogeneity of these compounds were checked by X-ray diffraction and thermomagnetic measurements. Lattice parameters were measured 
using X-ray powder diffractometer Siemens 5000 with $\mathrm{Cu} K_{\alpha}$ radiation. Recorded lines were fitted with X-Profiler (written by A. Kachel) and the IIX61 programme was applied to find exact values of the lattice parameters.

Magnetic moment was studied at fields up to $5 \mathrm{~T}$ in the temperature range $1.7 \mathrm{~K}$ to room temperature. Magnetocrystalline anisotropy was investigated by means of ac susceptibility and torsion measurements in the temperature range $77-293 \mathrm{~K}$. The easy direction of magnetization (EMD) was determined from X-ray diffraction of aligned powders. The most of the samples showed the easy $c$-axis at room temperature. Thermomagnetic curves at high temperatures were measured using a Faradaid balance.

\section{Results and discussion.}

All investigated compounds have the tetragonal ThMn $\mathrm{Mn}_{12}$-type structure. Slight decrease in the $a$ and the $c$ parameters with the increase in Si content were observed but the Vegard law was not fulfilled (Fig. 1).

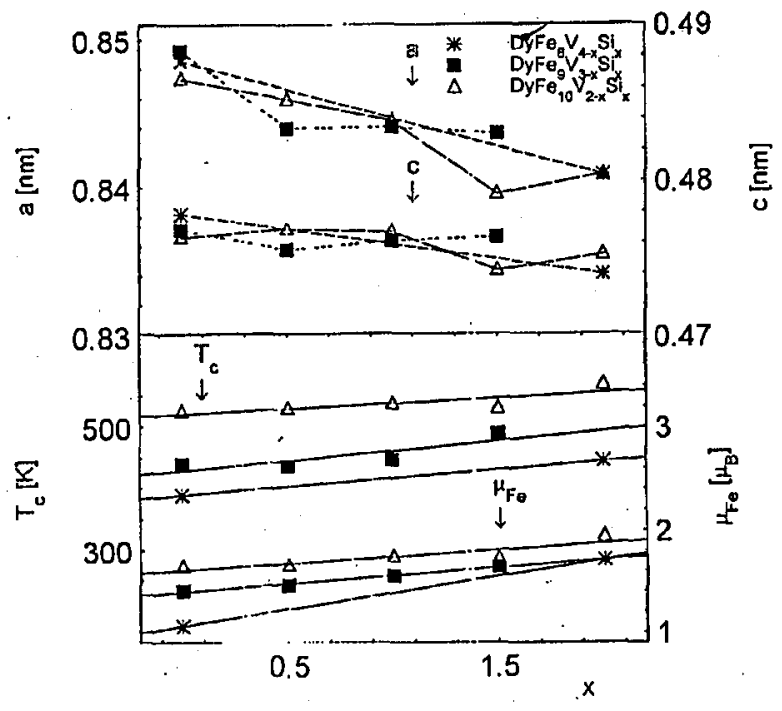

Fig. 1. Lattice parameters, $T_{c}$ and average $\mu(\mathrm{Fe})$ for $\mathrm{DyFe}_{12-x}(\mathrm{~V}, \mathrm{Si})_{x}$ versus Si concentration.

The investigated compounds are fcrimagnets with the Curie temperatures above room temperature. Plot of $T_{\mathrm{c}}$ as a function of silicon concentration is presented in Fig. 1. The values of $T_{\mathrm{c}}$ are almost unchanged for $\mathrm{DyFe}_{10} \mathrm{~V}_{2-x} \mathrm{Si}_{x}$ but increase in silicon-rich compounds of $\mathrm{DyFe}_{9} \mathrm{~V}_{3-x} \mathrm{Si}_{x}$ and $\mathrm{DyFe}_{8} \mathrm{~V}_{4-x} \mathrm{Si}_{x}$. The Curie temperature for $\mathrm{DyFe}_{8} \mathrm{~V}_{2} \mathrm{Si}_{2}$ is about $60 \mathrm{~K}$ higher than for $\mathrm{DyFe}_{8} \mathrm{~V}_{4}$. Moreover, as shown in Fig. 2, the resultant magnetization increases with the.Si content. Assuming that the magnetic moment of the Dy ions takes its free-ion value $\left(10 \mu_{\mathrm{B}}\right)$ the magnetic moment of the Fe atoms can be calculated from the values of the saturation magnetization obtained at $1.7 \mathrm{~K}$. The estimated moment on Fe atom increases 


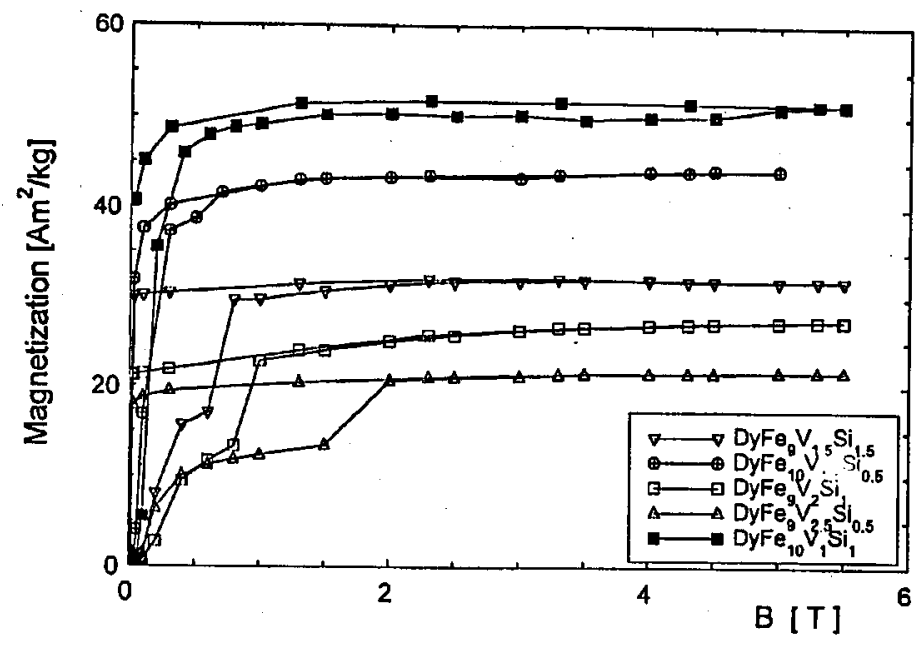

Fig. 2. Magnetic isotherms obtained at $1.7 \mathrm{~K}$.

markedly in $\mathrm{DyFe}_{12-x}(\mathrm{~V}, \mathrm{Si})_{x}$ systems when compared with $\mathrm{DyFe}_{12-x} \mathrm{~V}_{x}$. This is attributed to the enhancement of the exchange interaction due to the replacement of $\mathrm{V}$ by $\mathrm{Si}$ atoms. The effect of substituted $\mathrm{Si}$ atoms is similar to that observed in $\mathrm{DyFe}_{12-x}(\mathrm{~V}, \mathrm{C})_{x}$ under carbon doping [2].

The ac-initial magnetic susceptibility versus temperature curves of $\mathrm{DyFe}_{10} \mathrm{~V}_{2-x} \mathrm{Si}_{x}$ (Fig. 3) shows strong anomalies which are shifted towards higher tcmperatures with increase in $x$. These peaks of ac-susceptibility correspond to spin reorientation due to magnetocrystalline anisotropy competition between the $3 d$ and rare-earth sublattices. At room temperature the EMD is determined by the Fe sublattice but at low temperatures the negative Dy anisotropy contributions cause the spin reorientation transition. The torsion measurements confirm the tilting of the EMD from the $c$-axis with lowering temperature. A cone ordering of the magnetic moments is obscrved for all studied compositions at $77 \mathrm{~K}$. Generally, silicon content increases temperature of the begimning of the spin reorientation transition. The anisotropy constants reported for $\mathrm{YFe}_{10} \mathrm{~V}_{2}$ and $\mathrm{YFe}_{10} \mathrm{Si}_{2}$ are nearly the same [3]. It follows that magnetocrystalline anisotropy of dysprosium sublattice is enhanced because any essential changes in the $3 d$ sublattices are not expected.

The analysis of spin reorientation in compounds with lower concentration of iron (e.g. Dy $\mathrm{Fe}_{9} \mathrm{~V}_{3-x} \mathrm{Si}_{x}$ and $\mathrm{DyFe}_{8} \mathrm{~V}_{2} \mathrm{Si}_{2}$ ) is more complicated. It should be noticed that metamagnetic behavior of resultant magnetic moment is observed at low temperatures (Fig. 2). Changes in the magnetization under the action of an external field seem to indicate the spin-flip. It follows from Fig. 2 that the critical field values depend on vanadium and silicon content in $\mathrm{DyFe}_{12-x}(\mathrm{~V}, \mathrm{Si})_{x}$ series. The results of torsion measurements also demonstrate an influence of the applied magnetic field on the magnetic arrangement in the studied compounds. 


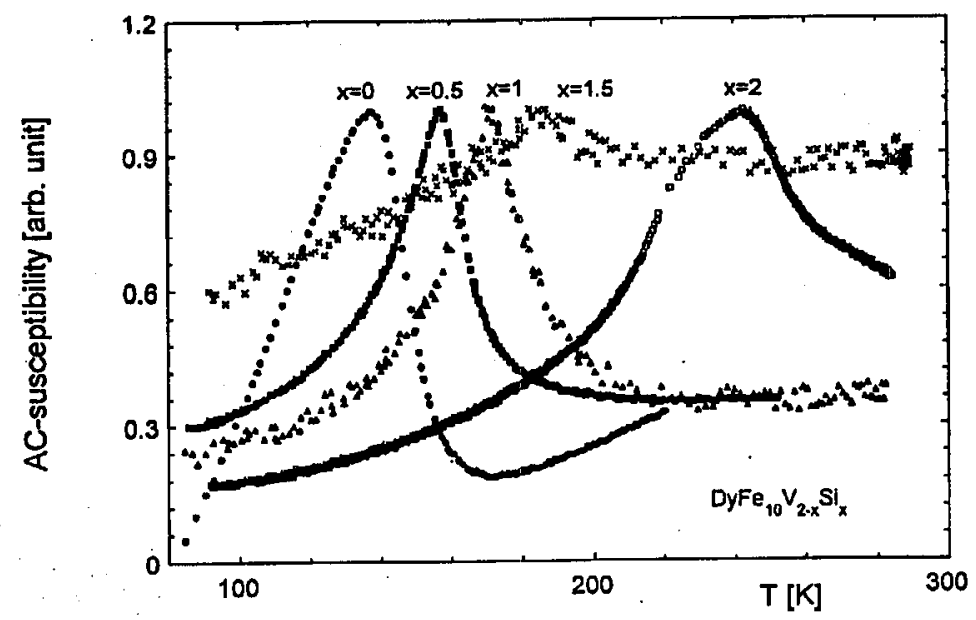

Fig. 3. ac-initial magnetic susceptibilities for series $\mathrm{DyFe} \mathrm{F}_{10} \mathrm{~V}_{2-x} \mathrm{Si}_{x}$.

The EMD in $\mathrm{DyFe}_{9} \mathrm{~V}_{3-x} \mathrm{Si}_{x}$ determined from torsion curves at $77 \mathrm{~K}$ in sufficiently small magnetic field is still the $c$-axis but markedly tilts from the $c$-axis in magnetic fields higher than $0.4 \mathrm{~T}$. Temperature and field dependence of the spin reorientation transition are due to the varying the nommagnetic $M$ element concentration.

The obtained results indicate that magnetic properties of $\mathrm{DyFe}_{12-x} \mathrm{M}_{x}$ depend on the stabilizing element $M$. In these compounds, three different crystallographic sites are available for the $\mathrm{Fe}$ and $\mathrm{M}$ atoms. The $\mathrm{V}$ atoms prefer the $8 \mathrm{i}$ sites [3] whereas the $\mathrm{Si}$ atoms share the $8 \mathrm{j}$ and $8 \mathrm{f}$ positions with the Fe atoms [4]. The deeper analysis taking into account the various distribution of the $\mathrm{Fe}$ and $\mathrm{M}$ atoms and its influence on the exchange and crystal field interactions responsible for magnetic behavior in the studied compounds will be done elsewhere.

\section{Acknowledgments}

Authors are indebted to Professor R. Troć for making possible the measurements of magnetization at $1.7 \mathrm{~K}$. This work is partly supported by grant No. 2 P30224403.

\section{References}

[1] W. Suski, in: Handbook on the Physics and Chemistry of Rare Earths, Vol. 22, Eds. K.A. Gschneidner, Jr., L. Eyring, Elsevier, Amsterdam 1996, Ch. 149, p. 143.

[2] Z. Drzazga, A. Winiarska, W. Zarek, E. Popiel, L. Gladczuk, J. Alloys Comp. 230, 76 (1995).

[3] M. Solzi, L. Pareti, O. Moze, W.I. Dawid, J. Appl. Phys. 64, 5084 (1988).

[4] C. Christides, M. Anagnostou, Ilong-Shuo Li, A. Kostikas, D. Niarchos, Phys. Rev. $B$ 44, 2182 (1991). 